\title{
Error assessment of lattice Boltzmann equation method for variable viscosity flows
}

\author{
Jianghui Chao ${ }^{1, \ddagger}$, Renwei Mei ${ }^{1, *, \dagger}$ and Wei Shyy ${ }^{2, \S}$ \\ ${ }^{1}$ Department of Mechanical and Aerospace Engineering, University of Florida, Gainesville, FL 32611, U.S.A. \\ ${ }^{2}$ Department of Aerospace Engineering, University of Michigan, Ann Arbor, MI 48109, U.S.A.
}

\begin{abstract}
SUMMARY
In lattice Boltzmann simulations, variable viscosity can complicate the truncation error analysis and create additional interaction between the truncation error and the boundary condition error. In order to address this issue, two boundary conditions for the lattice Boltzmann equation (LBE) simulations are used, including an exact, but narrowly applicable scheme previously proposed by Noble et al. (Phys. Fluids 1995; 7(1):203-209) and the popular bounce-back-on-link scheme. Using a 2-D laminar channel flow with a specified variable viscosity as a test case, it is shown that the boundary treatment error does not have a significant interaction with the truncation error associated with variable viscosity. The truncation error behaviour of the LBE for flows with variable viscosity is further investigated through a comparison between the LBE solution and the Navier-Stokes solution, showing that in the presence of strong variable viscosity the truncation error behaviour of the LBE solution is consistent with that of the Navier-Stokes solution, indicating that the LBE model closely matches the Navier-Stokes model for fluid flows with large viscosity variation. Copyright (C) 2006 John Wiley \& Sons, Ltd.
\end{abstract}

Received 24 March 2006; Revised 1 August 2006; Accepted 2 August 2006

KEY WORDS: lattice Boltzmann equation; variable viscosity; truncation error; boundary condition error; finite difference

\section{INTRODUCTION}

The lattice Boltzmann equation (LBE) method offers a meso-scale framework for fluid dynamics computations. It recovers the macroscopic fluid flow solution based on averaging of the particle distribution functions, obtained by solving the simplified form of the Boltzmann equation [1-4]. It is shown that the LBE method has been quite successful in the computations of complex fluid flow applications, such as interfacial dynamics [5, 6], turbulent flows [7] and porous media fluid flows [8].

\footnotetext{
*Correspondence to: Renwei Mei, Department of Mechanical and Aerospace Engineering, University of Florida, 231 MAE-A building, P.O. 116250, Gainesville, FL 32611-6250, U.S.A.

†E-mail: rwmei@ufl.edu

${ }^{\ddagger}$ E-mail: jhchao@ufl.edu

${ }^{\S}$ E-mail: weishyy@umich.edu
} 
Since large velocity gradients often accompany substantial viscosity variations due to either shear-thinning effects or turbulence models, errors associated with the variable viscosity model need to be addressed before exploring the accuracy for these problems. With variable viscosity such as for turbulent flows treated with the eddy viscosity models [9], or for fluids with flow-dependent properties, additional truncation error appears in the macroscopic equations derived from the LBE [10]. With the help of the truncation error analysis, computational accuracy for those flows can be further understood. The truncation error behaviour of the LBE with constant viscosity has been recently studied by Holdych et al. [11]. However, the truncation error for variable viscosity problems has not been studied systematically in the literature.

Like other computational models, the LBE method needs to address the wall boundary conditions [12]. In particular, the no-slip wall boundary condition in the LBE method is based on the bounce-back concept $[2,3,13]$. A lot of work has been done to extend this simple scheme to curved boundaries with second-order accuracy [12,14-17]. These methods can handle geometrical complexities easily; however, they cannot exactly recover the no-slip boundary condition [18] at the mesoscopic level [12]. This inconsistency gives rise to the boundary condition error. Although the no-slip boundary condition of the LBE has been derived from hydrodynamic conditions on walls by Noble et al. [19] and Inamuro et al. [20], these exact no-slip boundary schemes are incapable of handling geometrically complex boundaries because they require the computational nodes to coincide with the physical boundaries.

The original bounce-back scheme and other improved treatments have been successful in constant viscosity laminar flows with expected accuracy [2, 4, 12, 21]. However, for variable viscosity problems, the boundary condition error for no-slip walls may interact with the truncation error. Thus, besides the truncation error behaviour itself, whether the second-order accuracy of the bounce-back scheme can be maintained in the presence of the variable viscosity is another open question.

In this paper, these issues are investigated via a fully developed laminar channel flow with a specified variable viscosity. With the help of the finite difference analysis, the truncation error of the LBE with variable viscosity is investigated. Two different specified viscosity distributions, which lead to different boundary layer characteristics, are employed to examine the errors associated with the variable viscosity, in the LBE model. The exact solution of this channel flow exists and can be used for error analysis.

In what follows, the LBE method and the boundary conditions will first be reviewed. We then present the variable viscosity laminar channel flow equations along with the exact solution. Based upon the exact solution and the finite difference analysis, the error behaviours due to the variable viscosity and the boundary condition schemes will be assessed. To separate the error associated with the variable viscosity from that with the boundary treatment, both Noble et al.'s scheme [19], which is exact but restricted to straight boundaries, and bounce-back-on-link (BBL) scheme, which is not exact but can handle irregular geometries, will be employed.

\section{LBE METHOD AND WALL BOUNDARY CONDITIONS}

\subsection{LBE method}

The lattice Boltzmann equation with the singer-relaxation-time (SRT) Bhatnagar-Gross-Krook (BGK) model can be written as [22,23]

$$
f_{\alpha}\left(\mathbf{x}_{i}+\mathbf{e}_{\alpha} \delta t, t+\delta t\right)-f_{\alpha}\left(\mathbf{x}_{i}, t\right)=-\frac{1}{\tau}\left[f_{\alpha}\left(\mathbf{x}_{i}, t\right)-f_{\alpha}^{(\mathrm{eq})}\left(\mathbf{x}_{i}, t\right)\right]
$$


where $f_{\alpha}$ denotes $f\left(\mathbf{x}, \mathbf{e}_{\alpha}, t\right)$, which is the distribution function in the direction of the $\alpha$ th discrete velocity $\mathbf{e}_{\alpha}, f_{\alpha}^{(\mathrm{eq})}$ is the corresponding equilibrium distribution function in the discrete velocity space, $\tau=\lambda / \delta t, \lambda$ is the relaxation time, and $\mathbf{x}_{i}$ represents computational nodes in physical space. By using the Chapman-Enskog multi-scale expansion, Navier-Stokes equation can be recovered from the LBE (Equation (1)) with the leading truncation error of $O\left[(\delta x)^{2},(\delta u)^{3}\right][10]$.

The most popular lattice model for simulating two-dimensional flows is the nine-velocity square lattice model, which is often referred to as the 2-D 9-velocity (D2Q9) model [24]. In this lattice model, the discrete velocities $\left(\mathbf{e}_{\alpha}\right)$ are as follows:

$$
\begin{aligned}
& \mathbf{e}_{0}=0 \\
& \mathbf{e}_{\alpha}=c(\cos ((\alpha-1) \pi / 4), \sin ((\alpha-1) \pi / 4)) \quad \text { for } \alpha=1,3,5,7 \\
& \mathbf{e}_{\alpha}=\sqrt{2} c(\cos ((\alpha-1) \pi / 4), \sin ((\alpha-1) \pi / 4)) \quad \text { for } \alpha=2,4,6,8
\end{aligned}
$$

where $c=\delta x / \delta t$. The corresponding equilibrium distribution functions are defined as

$$
f_{\alpha}^{(\mathrm{eq})}=\rho w_{\alpha}\left[1+\frac{3}{c^{2}} \mathbf{e}_{\alpha} \cdot \mathbf{u}+\frac{9}{2 c^{2}}\left(\mathbf{e}_{\alpha} \cdot \mathbf{u}\right)^{2}-\frac{3}{2 c^{2}} \mathbf{u} \cdot \mathbf{u}\right], \quad \alpha=0,1, \ldots, 8
$$

where $w_{\alpha}$ is the weight coefficient given by

$$
w_{0}=\frac{4}{9}, \quad w_{1}=w_{3}=w_{5}=w_{7}=\frac{1}{9}, \quad w_{2}=w_{4}=w_{6}=w_{8}=\frac{1}{36}
$$

The density and momentum fluxes can be obtained from the moments of the distribution function as

$$
\rho=\sum_{\alpha=0}^{8} f_{\alpha}=\sum_{\alpha=0}^{8} f_{\alpha}^{(\mathrm{eq})}, \quad \rho \mathbf{u}=\sum_{\alpha=0}^{8} \mathbf{e}_{\alpha} f_{\alpha}=\sum_{\alpha=0}^{8} \mathbf{e}_{\alpha} f_{\alpha}^{(\mathrm{eq})}
$$

The kinematic viscosity associated with the D2Q9 lattice model can be expressed as

$$
v=\left(\tau-\frac{1}{2}\right) c_{s}^{2} \delta t
$$

where $c_{s}$ is the speed of sound, which is equal to $c / \sqrt{3}$ for D2Q9 lattice model. The corresponding equation of state is $p=\rho c_{s}^{2}$.

For a hexagonal lattice model [19], the discretized velocities are

$$
\begin{aligned}
& \mathbf{e}_{0}=0 \\
& \mathbf{e}_{\alpha}=c(\cos ((\alpha-1) 2 \pi / 6), \sin ((\alpha-1) 2 \pi / 6)) \text { for } \alpha=1,2, \ldots, 6
\end{aligned}
$$

The corresponding equilibrium distribution functions are

$$
\begin{aligned}
& f_{0}^{(\mathrm{eq})}=d_{0}-\frac{\rho}{c^{2}}(\mathbf{u} \cdot \mathbf{u}) \\
& f_{\alpha}^{(\mathrm{eq})}=\frac{\rho-d_{0}}{b}+\frac{\rho D}{c^{2} b}\left(\mathbf{e}_{\alpha} \cdot \mathbf{u}\right)+\frac{\rho D(D+2)}{2 c^{4} b}\left(\mathbf{e}_{\alpha} \cdot \mathbf{u}\right)^{2}-\frac{\rho D}{2 c^{2} b}(\mathbf{u} \cdot \mathbf{u}) \quad \text { for } \alpha=1,2, \ldots, 6
\end{aligned}
$$


where for the 2-D hexagonal lattice model, the dimension rank is $D=2$, the number of lattice direction is $b=6$, the average rest particle density is $d_{0}=\rho / 2$. The kinematic viscosity for 2-D hexagonal lattice model is

$$
v=\left(\frac{2 \tau-1}{8}\right) \frac{\delta x^{2}}{\delta t}
$$

\subsection{Wall boundary conditions}

Equation (1) is a first-order difference equation for which boundary conditions for $f_{\alpha}$ are needed. Among various approximations, the bounce-back boundary scheme is the most popular one. However, the bounce-back scheme results in a slip wall velocity [18], which increases with the relaxation time [19]. Different bounce-back schemes have been proposed so far. For example, if the computational nodes are located on the wall, it is referred to as bounce-back-on-node (BBN) scheme. Whereas, if the boundary is shifted into fluid side by one half lattice unit, i.e. placing the wall between computational nodes as shown in Figure 1, second-order accuracy can be achieved by bounce-back scheme [4, 14, 15, 25]; this is referred to as bounce-back-on-the-link (BBL) scheme [14]. For arbitrary curved walls with no-slip boundaries, second-order schemes have been proposed $[4,9,12,17]$.

Noble et al. developed an exact no-slip wall condition for hexagonal lattice [19]. This boundary scheme was derived from macroscopic mass and momentum conservation on a no-slip boundary. However, in this method, the boundary nodes have to be located exactly on walls. This restriction prevents the scheme from being used for flows with complex geometries. Noble et al.'s scheme is illustrated in Figure 2. For the boundary that is parallel to $\mathbf{e}_{1}$ and $\mathbf{e}_{4}$ shown in Figure 2, the following expressions for the unknown distribution functions $f_{2}$ and $f_{3}$ and unknown density $\rho$ at boundary nodes can be obtained by solving density and mass flux from the boundary nodes (see details in Reference [19])

$$
\begin{aligned}
& f_{2}+f_{3}=\rho-\left(f_{0}+f_{1}+f_{4}+f_{5}+f_{6}\right) \\
& f_{2}-f_{3}=2 \rho u-\left(2 f_{1}-2 f_{4}-f_{5}+f_{6}\right) \\
& f_{2}+f_{3}=(2 / \sqrt{3}) \rho v+\left(f_{5}+f_{6}\right)
\end{aligned}
$$

The standard BBL scheme and Noble et al.'s scheme (10) will be used in the present work to assess the computational errors associated with variable viscosity.

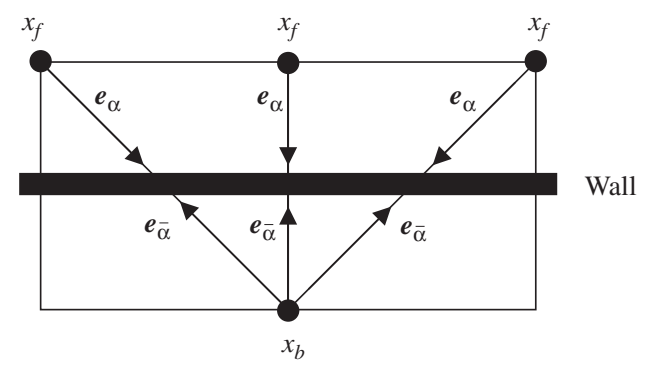

Figure 1. Boundary nodes and their neighbours using the square lattice. 


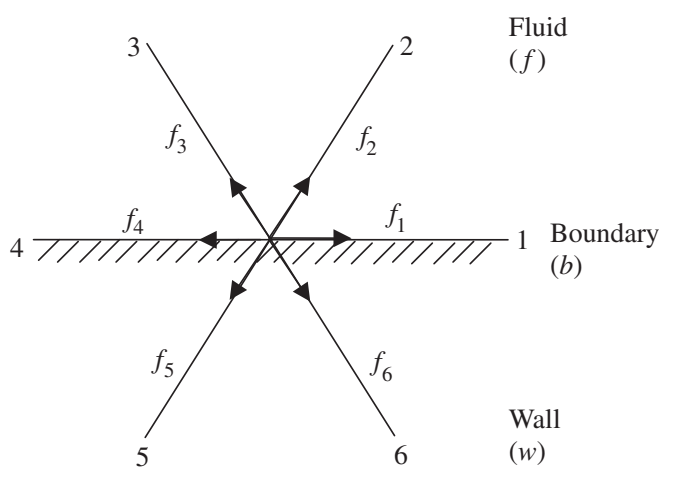

Figure 2. A boundary cell using the hexagonal (FHP) lattice [19].

\section{RESULTS AND DISCUSSION}

\subsection{Fully developed laminar channel flow with variable viscosity}

The governing equation of a 2-D fully developed steady laminar flow with variable viscosity in a channel of height $H$ is

$$
-\frac{1}{\rho} \frac{\mathrm{d} p}{\mathrm{~d} x}+\frac{\mathrm{d}}{\mathrm{d} y}\left(v_{\text {total }} \frac{\mathrm{d} u}{\mathrm{~d} y}\right)=0 \quad \text { and }\left.\quad u\right|_{y=0}=0,\left.\quad \frac{\mathrm{d} u}{\mathrm{~d} y}\right|_{y=H / 2}=0
$$

The viscosity $v_{\text {total }}$ is modelled as

$$
\begin{aligned}
v_{\text {total }} & =v_{0}+v_{\mathrm{st}} \\
\frac{v_{\mathrm{st}}}{v_{0}} & =\frac{\eta^{2}}{\theta^{2}\left(1+\eta^{3} / \delta\right)}=\frac{(\eta / \theta)^{2}}{\left[1+(\eta / \theta)^{3} \cdot \theta^{3} / \delta\right]}, \quad 0 \leqslant \eta=\frac{y}{H} \leqslant 0.5
\end{aligned}
$$

where $v_{0}$ is a constant and the symmetry gives $v_{\text {total }}$ for $0.5<\eta \leqslant 1$. The two dimensionless parameters $\delta$ and $\theta$ control the profile shapes for viscosity and velocity. Employing the boundary conditions and integrating Equation (16) twice yield

$u(\eta)=\frac{H^{2}}{\rho v_{0}} \frac{\mathrm{d} p}{\mathrm{~d} x}\left\{\begin{array}{c}\frac{1}{2}\left(\eta^{2}-\eta\right)-D \eta+A \ln (\eta / a+1)+\frac{B}{2}\left[\ln \left(\eta^{2}+b \eta+c\right)-\ln (c)\right] \\ +\left(Q-\frac{1}{2} b B\right) \frac{2}{\sqrt{4 c-b^{2}}}\left[\tan ^{-1}\left(\frac{2 \eta+b}{\sqrt{4 c-b^{2}}}\right)-\tan ^{-1}\left(\frac{b}{\sqrt{4 c-b^{2}}}\right)\right]\end{array}\right\}$

The derivation and the definitions of the parameters in Equation (13) are given in Appendix A.

In order to explore the truncation error due to variable viscosity under the condition of large velocity gradient over a short distance, the parameters $\delta$ and $\theta$ in Equation (12) need to be chosen carefully so that boundary-layer like velocity profile can be obtained in Equation (13). The first term in Equation (13) in the curly bracket corresponds to the parabolic velocity profile with the constant viscosity $v_{0}$. Other terms are due to variable part of the viscosity $v_{\mathrm{st}}$. For small values of $\delta$ and $\theta$, the fourth term $B / 2\left[\ln \left(\eta^{2}+b \eta+c\right)-\ln (c)\right]$ does not change dramatically across the 


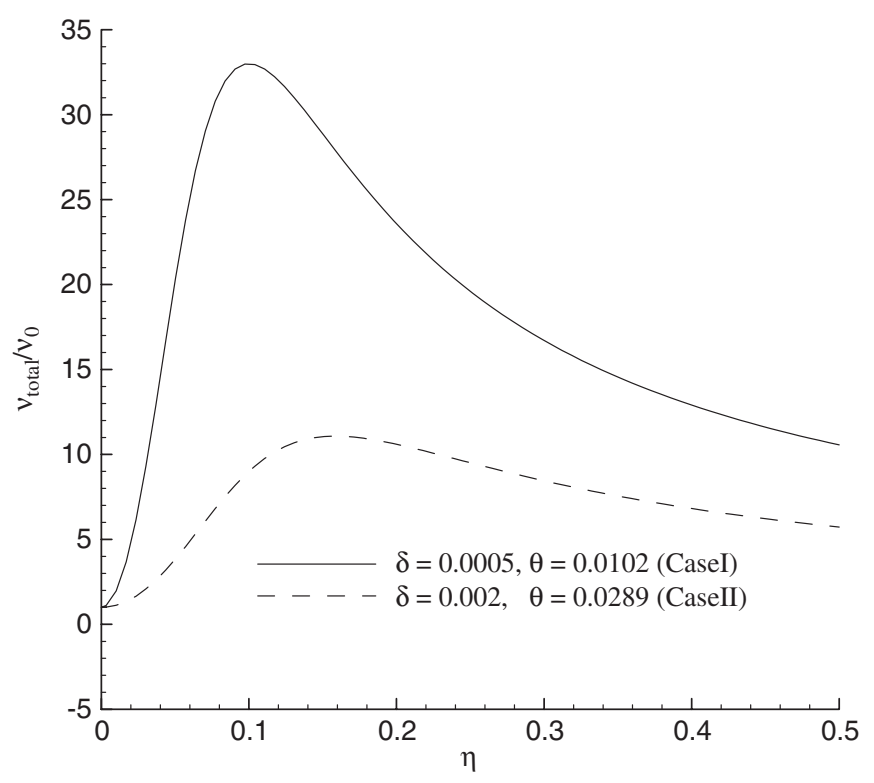

Figure 3. Two set of viscosity distributions used in this study.

whole channel. The second term $-D \eta$ is a negative linear term and the third term $A \ln (\eta / a+1)$ is positive across the channel, the sum of these two terms varies slowly in the near wall region. The last term in the near wall region varies rapidly because, for small $\delta$ and $\theta$, under further assumption of

$$
\theta \gg \delta^{3 / 2}
$$

it is asymptotically equal to

$$
\left(Q-\frac{1}{2} b B\right) \frac{2}{\sqrt{4 c-b^{2}}}\left[\tan ^{-1}\left(\frac{2 \eta+b}{\sqrt{4 c-b^{2}}}\right)-\tan ^{-1}\left(\frac{b}{\sqrt{4 c-b^{2}}}\right)\right] \approx-\frac{1}{2} \theta \tan ^{-1}\left(\frac{\eta}{\theta}\right)
$$

Thus, with small values of $\delta$ and $\theta$, the $\tan ^{-1}(.$.$) term in Equation (13) results in the boundary$ layer type of behaviour.

For comparison purpose, two sets of viscosity distributions, which satisfy Equation (14) for small $\delta$ and small $\theta$, are used in this study:

Case I: $\left(v_{0}, \delta, \theta\right)=(0.004167,0.0005,0.0102)$

Case II: $\left(v_{0}, \delta, \theta\right)=(0.008333,0.002,0.0289)$

The corresponding viscosity profiles are shown in Figure 3 . The resulting velocity distributions corresponding to these two viscosity distributions are shown in Figure 4. The boundary layer effect from the $\tan ^{-1}(.$.$) terms in Equation (13) or Equation (15) for Case I is shown in the inset$ of Figure 4. Equation (15) also gives a guideline for estimating the grid resolution required for resolving the boundary layers. Since $\tan ^{-1}(1) / \tan ^{-1}(\infty)=1 / 2$, it is seen that over a distance of 


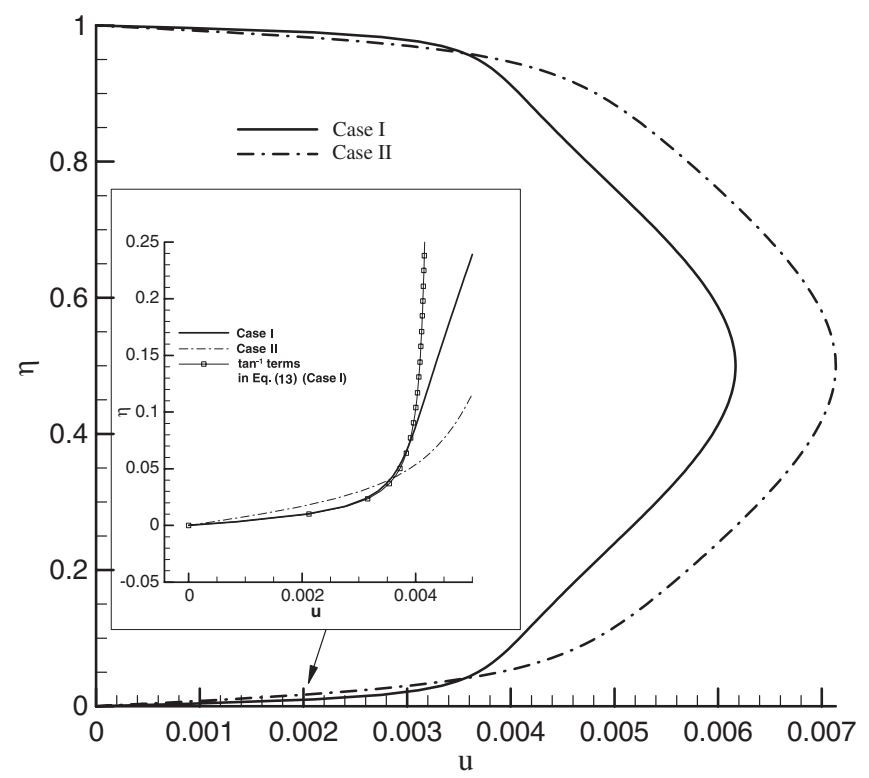

Figure 4. The exact velocity profiles of the channel flows with different boundary layer thicknesses due to different viscosity distributions. The parameters are: $\left(v_{0} ; \delta ; \theta\right)=(0.004167 ; 0.0005 ; 0.0102)$ for Case I and $\left(v_{0} ; \delta ; \theta\right)=(0.008333 ; 0.002 ; 0.0289)$ for Case II.

$\eta=\theta$, the velocity reaches $50 \%$ of the maximum given by Equation (15). Thus, for dimensionless grid size $h=1 / H$ that is close to or larger than $\theta$, numerical solutions will not have sufficient resolution for this thin layer.

\subsection{The lattice Boltzmann equation treatment}

In LBE method, the viscosity is associated with the relaxation time $\tau$ by Equation (6). The variable viscosity in LBE can still be realized via a spatially varying relaxation time, and the total viscosity at a given location can be expressed as

$$
\begin{array}{ll}
v_{\text {total }}(x, y)=\frac{2 \tau(x, y)-1}{6} & \text { for square grid } \\
v_{\text {total }}(x, y)=\frac{2 \tau(x, y)-1}{8} & \text { for hexagonal grid }
\end{array}
$$

Thus, the relaxation time can be represented by the local fluid viscosity as

$$
\begin{array}{ll}
\tau(x, y)=\frac{6 v_{\text {total }}(x, y)+1}{2} & \text { for square grid } \\
\tau(x, y)=\frac{8 v_{\text {total }}(x, y)+1}{2} & \text { for hexagonal grid }
\end{array}
$$

The computational set-up for BBL scheme is shown in Figure 5. The first computational node is half a lattice away from the channel wall. For Noble et al.'s scheme, the set-up is the same 


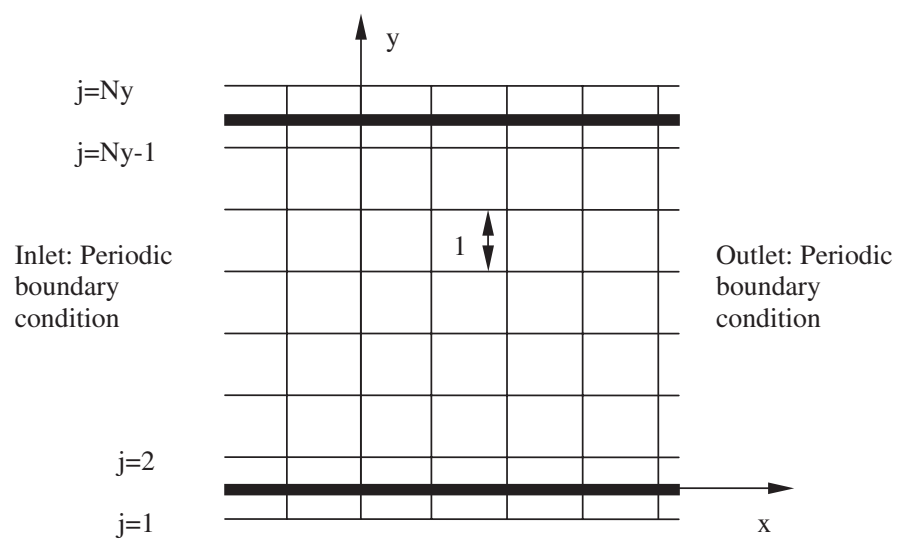

Figure 5. Square lattice distribution in channel flow simulation.

except that the grid is hexagonal and the computational boundary nodes are on the channel walls. For comparison purpose, the computations on hexagonal grid and square grid should have the same grid resolution across the channel height. For the same grid resolution the channel height of the square grid setup is $2 / \sqrt{3}$ times that of the hexagonal grid setup. In order to make absolute comparison among the velocity profiles, the pressure gradients are accordingly adjusted in each computation so that $H^{2}(\mathrm{~d} p / \mathrm{d} x)$ remains the same. Periodic boundary condition is used at the left and right side boundaries. The constant pressure gradient in Equation (11) is treated as a body force, and is added to the distribution functions after the collision step [21]. The error analysis for the computations with both boundary conditions is carried out by examining the difference between the exact solution and the computational results at each $y$ location.

\subsection{Assessment}

Since Case I exhibits thinner boundary layers and sharper near-wall velocity gradients than Case II, we first discuss Case I. Figure 6 compares three velocity profiles for $H=50(h=1 / H=0.02)$ : (a) exact solution; (b) LBE solution using the standard BBL boundary condition and square lattice formulation; (c) LBE solution using Nobel et al.'s exact boundary condition and hexagonal lattice formulation. For the parameters considered, $H=50(h=0.02>\theta=0.0102)$ does not resolve the thin boundary layer near the wall, as clearly shown in the inset of Figure 6. It is noted that due to the exactness of the boundary condition used, the hexagonal formulation gives only a slight overshoot in the velocity one full lattice away from the wall (at $h / \theta \sim 2$ ); however, it results in an overshoot for the rest of the lattices in the channel. The LBE solution using square lattice formulation suffers from both the inaccuracy of the bounce-back boundary condition and the insufficient near wall resolution and gives lower velocity throughout the entire channel. Both LBE velocity profiles have errors of similar magnitudes. This implies that when the velocity in the boundary layer is not sufficiently resolved, the exact boundary condition with the hexagonal lattice formulation would not offer any advantage compared to the approximate BBL condition using the square lattice formulation. For $h$ ranging from 0.004 to $0.1(H: 10-250$ in lattice unit) the square lattice formulation with the BBL boundary scheme shows different over/under-predictions 


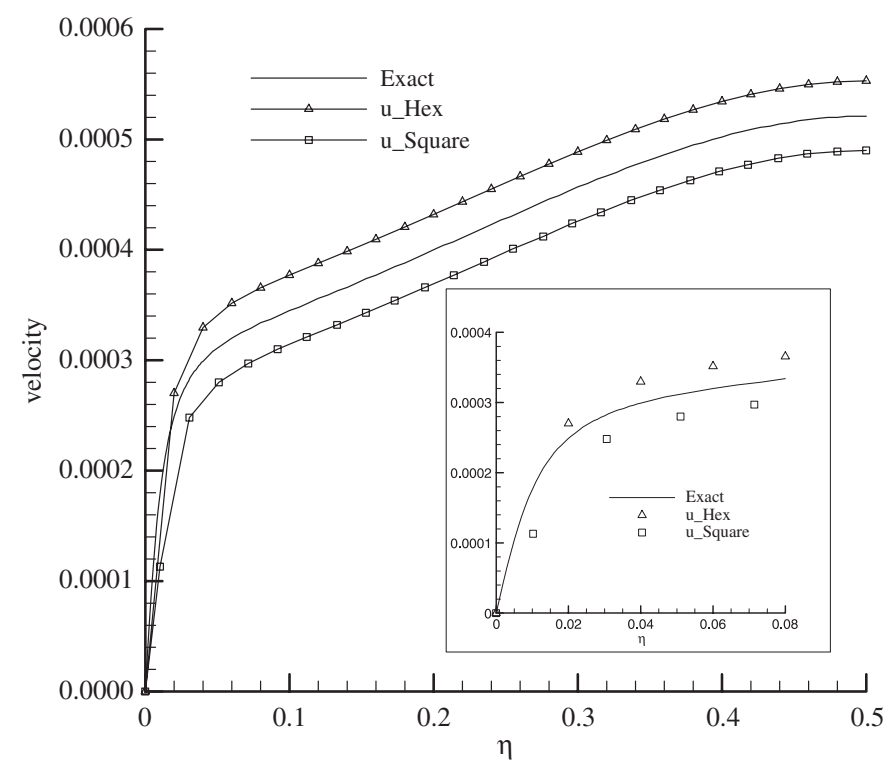

Figure 6. Comparison of the LBE velocity profiles using square lattice (with bounce-back on the link boundary condition) and hexagonal lattice (with Nobel et al.'s exact boundary condition) with the exact solution at $H=50$ lattice units.

for the velocity profiles from the hexagonal formulation comparing with Noble et al.'s scheme. While the results are not shown here for brevity, it suffices to note that the magnitudes of the over-predictions in the hexagonal lattice case are comparable to those of the under-predictions in the square lattice case. These velocity profiles indicate that the errors of the LBE solution using both boundary condition schemes are comparable. Thus, the relative $L_{2}$-norm error of the LBE solution, defined as

$$
E_{2}=\frac{\left\{\int_{0}^{H}\left[u_{\text {LBM }}(y)-u_{\text {exact }}(y)\right]^{2} \mathrm{~d} y\right\}^{1 / 2}}{\left[\int_{0}^{H} u_{\text {exact }}^{2}(y) \mathrm{d} y\right]^{1 / 2}}
$$

is examined for both boundary condition schemes.

The LBE computations with these two sets of viscosity distributions are carried out by using both Noble $e t$ al.'s scheme and the BBL scheme for grid size $h$ ranging from 0.004 to 0.1 . Their relative $L_{2}$-norm errors in the velocity profiles with respect to grid resolution are shown in Figure 7 (a). As expected, the relative $L_{2}$-norm error curve of Case I shifts up with respect to that of Case II because Case II has a thicker boundary layer which implies better computational resolution than in Case I for the same $h(=1 / H)$. For sufficiently high grid resolution $(h<0.01$, points A-D shown in Figure 7(a)), both Noble et al.'s scheme and the BBL scheme yield the asymptotic second-order accuracy, which is consistent with the truncation error analysis for both square and hexagonal lattice schemes.

For the present fully developed 2-D channel flow with low speed, the velocity field satisfies $\nabla \cdot u=0$. Thus the errors in LBE computations are mainly from the truncation error (due to 

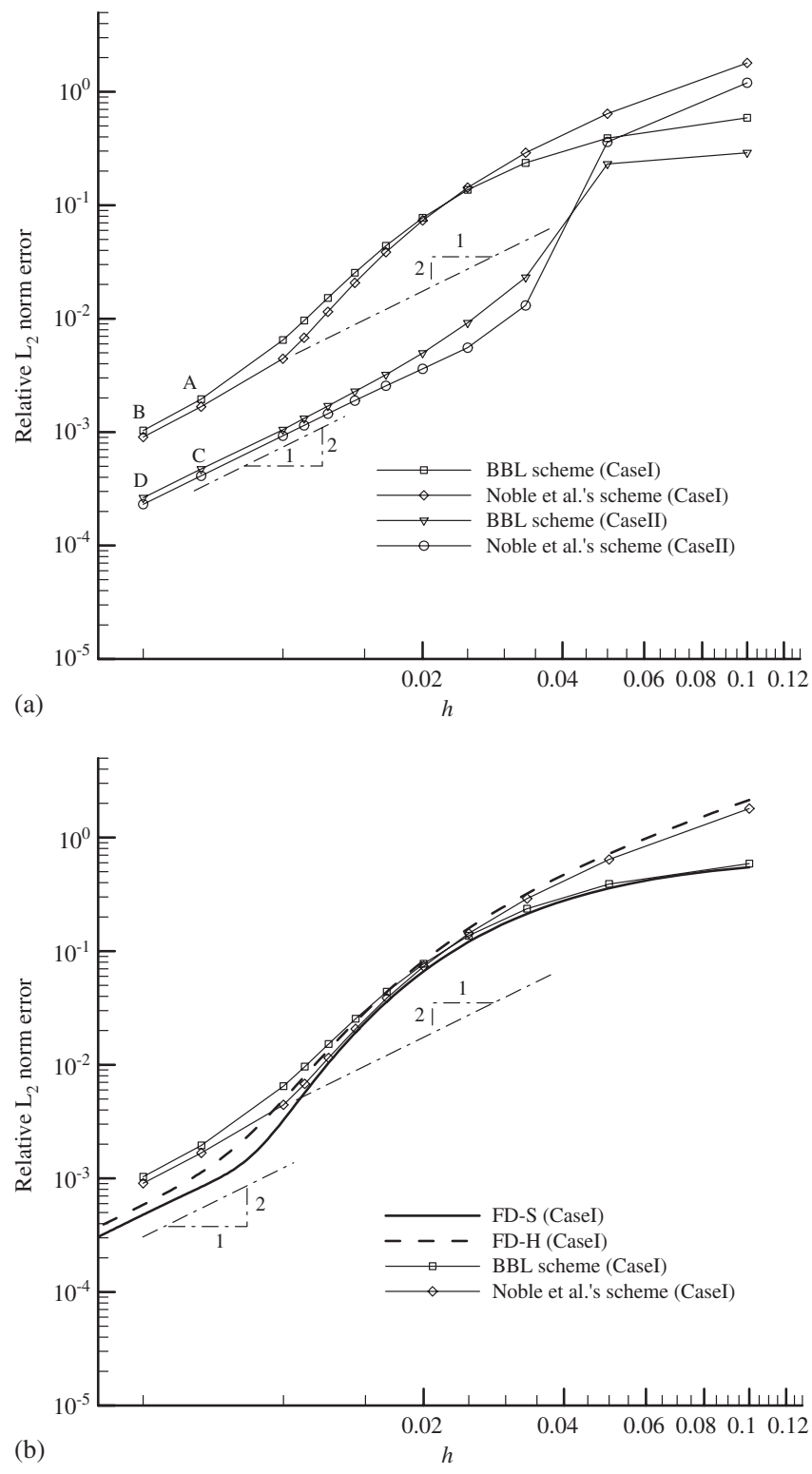

Figure 7. Dependence of the relative $L_{2}$-norm error on the lattice size $h$ in the fully developed channel flow with variable viscosity. The viscosity parameters are: $(\delta, \theta)=(0.0005,0.0102)$ for Case I, $(0.002,0.0289)$ for Case II. (a) The LBE with the boundary conditions of Noble et al.'s scheme and BBL scheme for both Case I and Case II. (b) The finite difference and the LBE with boundary conditions of Noble et al.'s scheme and BBL scheme for Case I.

variable viscosity) and the boundary condition treatment. When there is sufficient grid resolution (points A-D in Figure 7(a)), the relative $L_{2}$ norm errors of BBL scheme are about $15 \%$ larger than those of Noble et al.'s scheme. Since Noble et al.'s scheme does not contain boundary 
condition-induced error, this $15 \%$ difference in error in the BBL scheme results from the boundary condition treatment. Thus comparing the results using Noble et al.'s scheme and the BBL scheme, it can be inferred that in the presence of the strong velocity profile variation the truncation error contributes a significant part of the overall computational error.

Because a substantial part of the overall error is from the truncation error as opposed to the boundary condition error with highly variable viscosity or strongly varying velocity profile, the truncation error associated with the variable viscosity thus deserves close attention. However, the truncation error could have very complex form, even for the problems with constant viscosity [11]. It is recognized that there is a close relation between the LBE and the finite difference form of the momentum transport represented by the Navier-Stokes equation [18, 26, 27]. For example, He et al. [18] showed that the square lattice formulations for the particle distribution functions in a 2-D pressure driven channel flow with constant viscosity, after averaging, leads to a second order, central-difference formulation for the axial velocity. For the present channel flow problem with the large variation in viscosity, such derivation could not be easily obtained. However, if the relative $L_{2}$-norm error of the finite difference method still behaves similarly to that of the LBE, it is reasonable to expect that the truncation error behaviour of the LBE is similar to that of the finite difference-based macroscopic model (in the present case, the Navier-Stokes equation). This hypothesis will be assessed first.

In the Navier-Stokes model, the governing equation (Equation (11)) is discretized by standard central difference scheme (with $\Delta y=1$ lattice unit):

$$
0=-\frac{H^{2}}{\rho} \frac{\mathrm{d} p}{\mathrm{~d} x}(\Delta \eta)^{2}+v_{j+1 / 2}\left(u_{j+1}-u_{j}\right)-v_{j-1 / 2}\left(u_{j}-u_{j-1}\right)
$$

To compare the errors of the finite difference and LBE schemes, two finite difference solutions on different grid arrangements are obtained for the velocity profile. The first finite difference solution has the same grid arrangement as in the hexagonal lattice so that the first fluid node is one full mesh away from the wall. The second finite difference solution has the same grid arrangement as in the square lattice with the BBL scheme so that the first fluid node locates half a mesh away from the wall. This second finite difference solution requires an approximation for the velocity condition at the walls; a linear extrapolation is used in conjunction with the no-slip condition at the wall. For completeness, a second-order extrapolation is also used to approximate the derivative at the wall in solving Equation (11); the error is consistently larger than the linear extrapolation and hence the results are not presented. The viscosity distribution of Case I is chosen for the finite difference computations for Case I gives sharper near-wall velocity gradients than Case II.

The results of the relative $L_{2}$-norm errors over a range of $h=0.004$ to 0.1 from these two finite difference solutions are shown in Figure 7(b). The results labelled as 'FD-H' refer to the finite difference solution obtained on the hexagonal grid and the results labelled as 'FD-S' refer to the finite difference solution obtained on the square grid. For small values of $h(h<0.01)$, the $O\left(h^{2}\right)$ asymptotic behaviour in error is clearly visible, which is similar to the LBE cases. This asymptotic error behaviour is expected because the LBE scheme has second-order accuracy and the boundary conditions are, depending on the specific scheme chosen, either exact or second-order accurate, and the finite difference scheme with the central difference discretization gives global second-order accuracy. As $h$ increases close to or greater than 0.01 , both the finite difference and the LBE errors increase faster than $O\left(h^{2}\right)$. Since the velocity profile given by the exact solution has a thin layer of thickness $\theta=0.0102$, the error starts to increase more rapidly for $h>0.01$ 
when the resolution of the thin layer becomes inadequate. For $0.01<h<0.033$ where the numerical resolution is insufficient, all the error curves are quite close, indicating that the need for a better resolution of the boundary layer exceeds the need for a better boundary condition. One also notes that when $h$ exceeds the boundary layer thickness, truncation errors associated with high-order velocity gradients may not be small.

Comparing the relative $L_{2}$-norm errors of the finite difference solution with those of the LBE solution in Figure $7(\mathrm{~b})$, it is seen that the relative $L_{2}$-norm error of 'FD-H' follows that of the LBE with Noble et al.'s scheme closely, and the relative $L_{2}$-norm error 'FD-S' follows that of the LBE with the BBL scheme closely. This consistent behaviour of the relative $L_{2}$-norm errors between both the LBE method and the finite difference method suggests that one could obtain an insight on the truncation error of the LBE schemes by studying the truncation error of its finite difference counterpart.

For both LBE and finite difference schemes, their modified equations associated with the corresponding truncation errors can be represented as

$$
\begin{gathered}
L\left(E_{\mathrm{LBE}}\right)=\mathrm{TE} \text { of the } \mathrm{LBE} \text { method } \\
L\left(E_{\mathrm{FD}}\right)=\mathrm{TE} \text { of the finite difference method }
\end{gathered}
$$

where $L$ is the differential operator $-\mathrm{d} / \mathrm{d} x\left(v_{\text {total }}(\mathrm{d} / \mathrm{d} x)\right), E_{\mathrm{LBE}}=u_{\text {exact }}-u_{\mathrm{LBE}}$, and $E_{\mathrm{FD}}=u_{\text {exact }}-$ $u_{\mathrm{FD}}$. Using Taylor series expansion the truncation error in Equation (21) is

$$
\mathrm{TE}=\left[\frac{1}{12} v_{\text {total }} \frac{\partial^{4} u}{\partial \eta^{4}}+\frac{1}{6} \frac{\partial v_{\text {total }}}{\partial \eta} \frac{\partial^{3} u}{\partial \eta^{3}}+\frac{1}{8} \frac{\partial v_{\text {total }}^{2}}{\partial \eta^{2}} \frac{\partial^{2} u}{\partial \eta^{2}}+\frac{1}{24} \frac{\partial v_{\text {total }}^{3}}{\partial \eta^{3}} \frac{\partial u}{\partial \eta}\right](\Delta \eta)^{2}+\text { HOT }
$$

Although the truncation error of the LBE is unknown, comparing the solution errors, $E_{\mathrm{FD}}$ and $E_{\mathrm{LBE}}$, on the left-hand sides of Equations (20) and (21) can offer insight into the truncation errors on the right-hand sides of these equations while this indirect comparison avoids the tedious derivation of the truncation error of the LBE. The value of $E_{\mathrm{FD}}$ can be computed by subtracting $u_{\mathrm{FD}}$ from the exact solution after the velocity $u_{\mathrm{FD}}$ is solved from Equation (19). To highlight the behaviour of the leading term of the truncation error, the values of $E_{\mathrm{FD}}$ are approximated by $E_{\mathrm{FD}}^{*}$ obtained from solving Equation (21) with small $h$. For $E_{\mathrm{LBE}}$ it can only be determined directly by subtracting $u_{\mathrm{LBE}}$ from the exact solution.

Equation (21) can be solved, with the boundary conditions $E_{\mathrm{FD}}(0)=E_{\mathrm{FD}}(1)=0$, by using the same central difference scheme given by Equation (19), and replacing the entire TE by the leading term of the TE provided that the resolution is sufficient and the high-order terms in the TE can be neglected. Caution must be exercised in interpreting the results from the numerical solution of Equation (21). In this equation, even if the leading term of the TE on the right-hand side can be analytically evaluated, the variation of the viscosity on the left-hand side is the source of the TE in the first place. When solving for $E(\eta)$ the effect of the TE associated with the ODE (Equation (21)) now is further compounded by the variation of the viscosity.

The finite difference computation for Equation (21) is carried out on a grid with the same grid arrangement and size as in the hexagonal lattice case so that the exact velocity boundary condition can be used. For Case I, the thin wall layer has a length scale $\theta=0.0102$ and can be adequately resolved using $\Delta \eta=1 / H=1 / 200$, which is confirmed by the comparison between $E_{\mathrm{FD}}$ and $E_{\mathrm{FD}}^{*}$ in Figure 8. The curve representing $E_{\mathrm{FD}}$ is almost on top of the curve representing $E_{\mathrm{FD}}^{*}$. Figure 8 also 


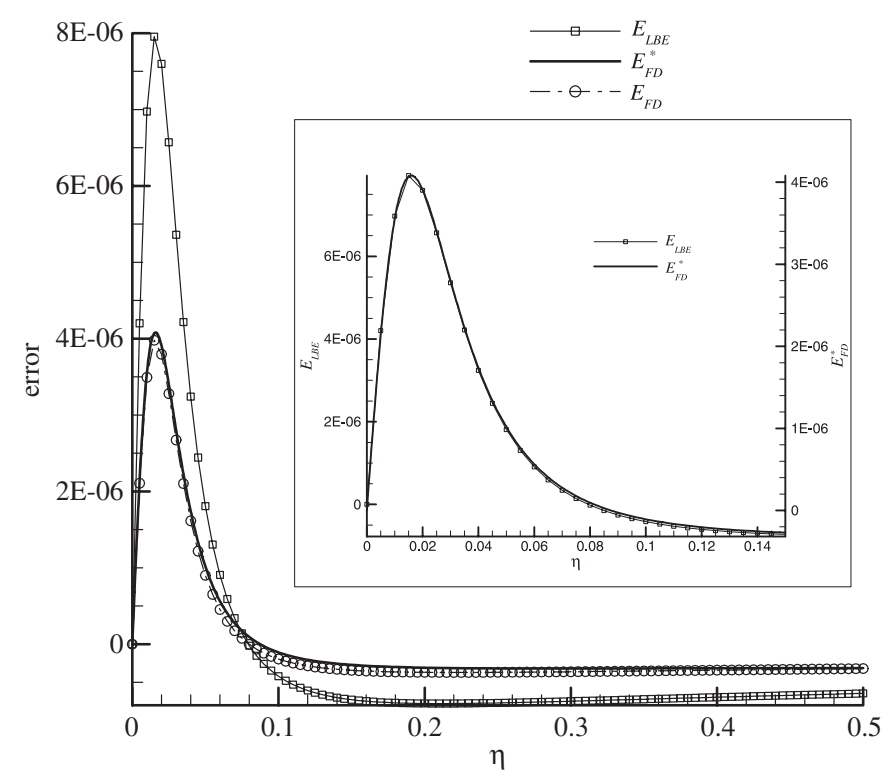

Figure 8. Comparison of $E_{\mathrm{LBE}}=u_{\text {exact }}-u_{\mathrm{LBE}}$ with $E_{\mathrm{FD}}=u_{\mathrm{exact}}-u_{\mathrm{FD}}$ for $H=200$, and $\delta=0.0005$, $\theta=0.0102, E_{\mathrm{FD}}^{*}$ is the numerical approximation of $E_{\mathrm{FD}}$ obtained from solving Equation (21).

shows the variation of $E_{\mathrm{LBE}}$ across the channel. Overall, $E_{\mathrm{FD}}^{*}$ from Equation (21) is smaller than $E_{\mathrm{LBE}}$ across the whole channel which implies that these two methods indeed have quantitatively different truncation errors. However, if we adjust the scale for $E_{\mathrm{FD}}^{*}$ by a factor of 2, these two curves lie almost on top of each other, as shown in the inset of Figure 8. The matching of the error curves between two solutions demonstrate that the LBE truncation error behaves very similarly to that observed in the finite difference scheme in the presence of a strong variation in the velocity profile. This is in agreement with the observation on the relative $L_{2}$-norm error behaviour shown in Figure 7(b).

Based on the foregoing discussions, it is seen that BBL scheme performs similarly as Nobel's scheme in the presence of strong velocity profile variation. In view of the simplicity and flexibility of the BBL scheme over the Noble et al.'s scheme and comparable performance in accuracy, it is attractive to use the BBL scheme (or its extended version such as Bouzidi et al. [17] and Yu et al. [4]) for handling problems of substantial velocity profile variations caused either by complex geometry or variable viscosity.

For complex 3-D flows with curved boundaries, Navier-Stokes solvers currently have more flexibility on grid arrangement. For example, body-fitted coordinates and grid stretching can be more easily implemented to improve grid resolution near boundaries than in the LBE solvers. Although the recent developments in LBE method such as multi-block [4] and composite grid [9] techniques can alleviate the difficulty in grid arrangement in LBE simulations to certain extent, further research efforts in LBE are needed. As the present study has indicated the boundary condition induced error may not be dominant, there is a great potential in improving the overall capability of the LBE solvers for complex flows with very strong velocity variation by focusing future research efforts on extending the grid flexibility of LBE schemes. 


\section{SUMMARY AND CONCLUSION}

In this paper, the error behaviour of the LBE method for a flow with strong variation in viscosity is studied. The variable viscosity in the LBE method is modelled through a variable relaxation time. Solutions for a laminar channel flow with a specified variable viscosity are obtained using both the LBE method and the finite difference method to examine the truncation error behaviour of the LBE method for flows with strong varying viscosity. The effect of the boundary condition error of the BBL scheme on the overall error is investigated via the comparison of the error behaviour of the BBL scheme and that of Noble et al.'s scheme. The results show that with rapid viscosity variation the boundary condition error of the BBL scheme does not induce noticeable, additional errors, and the overall error of such flows is dominated by the truncation error itself. The results also show that in the presence of strong variable viscosity the truncation error behaviour of the LBE solution is consistent with that of finite difference solution to Navier-Stokes equations.

\section{APPENDIX A: THE EXACT SOLUTION FOR THE CHANNEL FLOW}

Employing the boundary conditions in Equation (11), twice integrations of the governing equation yield

$$
u(\eta)=\frac{H^{2}}{\rho v} \frac{\mathrm{d} p}{\mathrm{~d} x} \int_{0}^{\eta} \frac{(\xi-0.5)\left(\xi^{3}+\delta\right)}{\xi^{3}+\frac{\delta}{\theta^{2}} \xi^{2}+\delta} \mathrm{d} \xi \quad \text { for } 0 \leqslant \xi \leqslant 0.5
$$

Let $\alpha=\delta / \theta^{2}$ and $\eta^{3}+\alpha \eta^{2}+\delta=(\eta+a)\left(\eta^{2}+b \eta+c\right)$. It is easily seen that $a^{3}-\alpha a^{2}=\delta, b=\alpha-a$ and $c=\delta / a$, where the value of $a$ can be obtained numerically in terms of $\alpha$ and $\delta$. Integrating Equation (A1) results in the exact solution of this laminar channel flow, that is,

$$
u(\eta)=\frac{H^{2}}{\rho v_{0}} \frac{\mathrm{d} p}{\mathrm{~d} x}\left\{\begin{array}{c}
\frac{1}{2}\left(\eta^{2}-\eta\right)-D \eta+A \ln (\eta / a+1)+\frac{B}{2}\left[\ln \left(\eta^{2}+b \eta+c\right)-\ln (c)\right] \\
+\left(Q-\frac{1}{2} b B\right) \frac{2}{\sqrt{4 c-b^{2}}}\left[\tan ^{-1}\left(\frac{2 \eta+b}{\sqrt{4 c-b^{2}}}\right)-\tan ^{-1}\left(\frac{b}{\sqrt{4 c-b^{2}}}\right)\right]
\end{array}\right\}
$$

where

$$
D=-\alpha, \quad A=\frac{\alpha\left[(1 / 2+\alpha) a^{2}+\delta\right]}{a^{2}+2 c}, \quad B=\frac{\alpha(1+2 \alpha-a)}{a\left(a^{2}+2 c\right)} \delta \quad \text { and } \quad Q=-(A b+B a)
$$

\section{REFERENCES}

1. McNamara GR, Zanetti G. Use of the Boltzmann equation to simulate lattice-gas automata. Physical Review Letter 1988; 61:2332-2335.

2. Chen S, Doolen GD. Lattice Boltzmann method for fluid flows. Annual Review of Fluid Mechanics 1998; 30:329-364.

3. Succi S. The Lattice Boltzmann Equation for Fluid Dynamics and Beyond. Oxford University Press: Oxford, 2001.

4. Yu D, Mei R, Luo L-S, Shyy W. Viscous flow computations with the method of lattice Boltzmann equation. Progress in Aerospace Sciences 2003; 39:329-367. 
5. He X, Chen S, Zhang R. A lattice Boltzmann scheme for incompressible multiphase flow and its application in simulation of Rayleigh-Taylor instability. Journal of Computational Physics 1999; 152:642-663.

6. Fan L, Fang H, Lin Z. Simulation of contact line dynamics in a two-dimensional capillary tube by the lattice Boltzmann model. Physical Review E 2001; 63(051603):1-6.

7. Yu H, Girimaji S, Luo L-S. Lattice Boltzmann simulations of decaying homogeneous isotropic turbulence. Physical Review E 2005; 71(016708):1-5.

8. Chen S, Diemer K, Doolen GD, Eggert K, Fu C, Gutman S, Travis BJ. Lattice gas automata for flow through porous media. Physica D 1991; 47:72-84.

9. Filippova Q, Hanel D. Grid refinement for lattice-BGK models. Journal of Computational Physics 1998; 147: 219-228.

10. Hou S, Zou Q, Chen S, Doolen GD, Cogley AC. Simulation of cavity flow by the lattice Boltzmann method. Journal of Computational Physics 1995; 118:329-347.

11. Holdych DJ, Noble D, Georgiadis J, Buckius R. Truncation error analysis of lattice Boltzmann methods source. Journal of Computational Physics 2004; 193:595-619.

12. Mei R, Luo L-S, Shyy W. An accurate curved boundary treatment in the lattice Boltzmann method. Journal of Computational Physics 1999; 155:307-330.

13. Lavallee P, Boon J, Noullez A. Boundaries in lattice gas flows. Physica D 1991; 47:233-240.

14. Ladd AJC. Numerical simulation of particular suspensions via a discretized Boltzmann equation. Part 1. Theoretical foundation. Journal of Fluid Mechanics 1994; 271:285-309.

15. Ladd AJC. Numerical simulation of particular suspensions via a discretized Boltzmann equation. Part 2. Numerical results. Journal of Fluid Mechanics 1994; 271:311-339.

16. Yu D, Mei R, Shyy W. A unified boundary treatment in lattice Boltzmann method. AIAA Paper New York 2003; 2003-0953.

17. Bouzidi M, Firdaouss M, Lallemand P. Momentum transfer of a Boltzmann-lattice fluid with boundaries. Physics of Fluids 2001; 13(11):3452-3459.

18. He X, Zou Q, Luo L-S, Dembo M. Analytic solutions of simple flow and analysis of non-slip boundary conditions for the lattice Boltzmann BGK model. Journal of Statistical Physics 1997; 87:115-136.

19. Noble DR, Chen S, Georgiadis JG, Buckius RO. A consistent hydrodynamic boundary condition for the lattice Boltzmann method. Physics of Fluids 1995; 7(1):203-209.

20. Inamuro T, Yoshino M, Ogino F. A non-slip boundary condition for lattice Boltzmann simulations. Physics of Fluids 1995; 7(12):2928-2930.

21. Mei R, Shyy W, Yu D, Luo L-S. Lattice Boltzmann method for 3-D flows with curved boundary. Journal of Computational Physics 2000; 161:680-699.

22. He X, Luo L-S. Theory of the lattice Boltzmann method: from the Boltzmann equation to the lattice Boltzmann equation. Physical Review E 1997; 56(6):6811-6817.

23. He X, Luo L-S. A priori derivation of the lattice Boltzmann equation. Physical Review E 1997; 55(6) R6333:1-4.

24. Qian Y, d'Humieres D, Lallemand P. Lattice BGK models for Navier-Stokes equation. Europhysics Letter 1992; 17(6):479-484.

25. Ziegler D. Boundary conditions for lattice Boltzmann simulations. Journal of Statistical Physics 1993; 71: 1171-1177.

26. He X, Doolen GD, Clark T. Comparison of the lattice Boltzmann method and the artificial compressibility method for Navier-Stokes equations. Journal of Computational Physics 2002; 179:439-451.

27. Mei R, Shyy W. On the finite difference-based lattice Boltzmann method in curvilinear coordinates. Journal of Computational Physics 1998; 143:426-448. 